XI. On the Chameleon's Retina; a further Contribution to the Minute Anatomy of the Retina of Reptiles. By J. W. Hulke, F.R.C.S., Assistant Surgeon to the Middlesex and Royal London Ophthalmic Hospitals. Communicated by William Bowas, F.R.S.

Received June 1,--Read June 15, 1865.

THE chameleon's retina is remarkable in two respects: 1st, it has a yellow spot and fovea centralis*; and 2ndly, the fibres which connect the cones with the inner layers cross the connective-tissue radial fibres instead of running parallel to them $\uparrow$.

The yellow spot lies in the centre of the retina, at the posterior pole of the globe. The fovea is a deep circular pit. Its bottom (i. e. centre) appears as a dark-brown dot, encircled by a lighter brown ring corresponding to the sides of the pit, and this in turn is surrounded by a colourless zone, the outer edge of which passes gradually into the colour of the surrounding parts (Plate XIX. fig. 1).

A flat subconical pecten, rising $\frac{1}{33}$ " above the inner surface of the retina, marks the entrance of the optic nerve, $1^{\prime \prime \prime}$, at the temporal side of the fovea. 'The nerve pierces the sclerotic very obliquely, and its bundles, escaping from the base of the pecten in the level of the ganglionic layer of the retina, are distributed on the inner retinal surface in such a manner that those only which belong to the fovea run directly towards this from the pecten, while all other fibres for parts on the nasal side of the pecten arch above and below the fovea in increasingly open curves.

\title{
Minute Structure.
}

1. Bacillary Layer.-Cones only occur in this layer (figs. $2 \& 3$ ). They consist of an inner and an outer segment, separated by a bright transverse line. The outer segment is a very slender, strongly-refracting shaft; it tapers slightly towards its outer end; its length increases from the periphery to the centre of the retina, but is always much less than that of the inner segment. It is completely buried in the choroid, which also sheaths the outer part of the inner segment. This latter segment, although much larger than the outer, is inconspicuous in fresh specimens in consequence of its low refraction. At the centre of the retina its length is very considerable, while its breadth scarcely exceeds that of the outer segment; these proportions give the cones in this part the appearance of tall slender pillars; but at the periphery the inner segment is stout, and the cones resemble tall flasks. In chromic acid preparations the outer part of the flask-shaped cone-body is finely granulated, while the inner is more homogeneous, and often exhibits

* Krox, Mem. Wern. Soc. vol. r. p. 2, and Edinb. Phil. Journal, Oct. 1823, p. 358.

† Müller, H., Würzb. Naturwiss. Zeitschr. 1s62, Bd. III. Heft 1. S. 20. 
an oval or stellate vacuole, regarded by MÜLLER as a nucleus, and which is not to be mistaken for an outer granule.

The difference between these extreme forms of cones in the centre and in the periphery of the retina is so great, that, judging from their shape alone, they might not be unreasonably regarded as different elements; but a regularly progressive change from one to the other shape in passing from the centre of the retina outwards, and the constant presence of a bright pale yellow or colourless bead in the outer end of their inner segment, establish their identity and conic nature. As their stoutness decreases from the periphery to the centre of the retina, each successive unit of surface in this direction contains more cones; and since each cone has its outer granule associated with it, the numerical increase of the cones is attended with a corresponding increase in the number of the outer granules. The inner ends of the cones pass through apertures in the membrana limitans externa, which in its structure and relations resembles that of other reptiles.

2. The Outer Granule-Layer.-The so-called "granules" are large, round, and roundlyoval nuclei, about $\frac{1}{6000}$ " in diameter. In the periphery of the retina, where the cones are stout, they lie in a single tier, one in the inner end of each cone, in or slightly inside the plane of the membrana limitans externa; while towards the fovea, where the slenderness of the cones does not allow them to enclose their outer granule, the granules form a separate layer, and each is connected with its cone by an intermediate fibre, continuous with the inner end of the latter. These fibres, for which I would suggest the term "primitive cone-fibres," are identical with those I have described as coming off from the inner end of the rods and cones in other reptiles, but their course and arrangement in the chameleon are peculiar.

3. Cone-fibre Plexus.-In the periphery of the retina, where the cones enclose their outer granule, the primitive fibres, on leaving the inner end of the cones, curve vertically inwards and combine in bundles, which run for a considerable distance horizontally towards the periphery, and then again bend vertically inwards among the inner granules. In the central region, where the cones are slender and numerous and the outer granules lie in several tiers, the primitive cone-fibres first bend inwards from the cones, next take up their outer granule, and then, at the inner surface of the outer granule-layer, combine in bundles which unite in a plexiform manner to form a layer that attains a remarkable thickness at the border of the fovea centralis. The inclination of the bundles, and their combination in the plexus, is such that the outer bundles are gradually brought to the inner surface of the plexus. Here they are again resolved into primitive fibres that curve inwards through the intergranule-layer to the inner granules (fig. $4 b$ ). H. MüLLER relates that in his hardened preparations he could not determine whether the finer fibres were merely stuck together in the bundles of the plexus, or whether divisions took place; my belief is that they cohere very closely in the bundles of the plexus, but that they never fuse or split. Lying between the outer and the inner granules, this plexus was called by MÜLLER the intergranule-layer, 
a name which I give to a thin band of connective tissue that bounds the outer surface of the inner granule-layer, and which corresponds to the intergranule-layer of other reptiles (fig. $5 i$ ).

A second set of fibres, corresponding to those which in the 'Turtle's retina I named the outer radial fibres, crosses the cone-fibre plexus nearly perpendicularly. These vertical radial fibres are most developed where the plexus is thickest (fig. 5). Near the fovea they are very conspicuous, particularly in carmine preparations, in which they are almost colourless, while the bundles of cone-fibres have a deep red tint. In sections parallel to the cone-fibre bundles we see the vertical radial fibres traversing the meshes of the plexus; while in sections across the bundles, the cut ends of these are packed in long vertical spaces between the radial fibres, which last appear as stout vertical pillars that divide at acute angles, and becoming fine and numerous, end at the inner surface of the membrana limitans externa. Near the inner surface of the plexus, the stout pillar-like fibres also repeatedly divide, the resulting fibrillæ spread in a fanlike manner, and interweaving with others from neighbouring trunks, form the thin band which I call the intergranule-layer. Through this layer the primitive cone-fibres pass to the inner granules.

The above description of the outer vertical radial fibres and intergranule-layer applies particularly to the central region of the retina; in the periphery, where the retina is thin, a distinct intergranule-layer is not present, and the outer vertical radial fibres are replaced by vertical radial fibres which pass from the inner to the outer limiting membrane.

4. The Layer of Inner Granules.-This layer throughout the retina is thicker than that of the outer granules. It reaches its greatest development around the fovea centralis, and diminishes towards the periphery in a more gradual manner than the outer granule-layer. Several distinct forms of cells and nuclei, and two sets of fibres, a vertical and an oblique, are distinguishable in it. Near the outer surface of the layer, in the central region in close relation to the vertical set of fibres just mentioned, we find small oat-shaped nuclei which carmine scarcely stains. Besides these, other minute nuclei, bright, angular, and also scarcely tinted by carmine, are scattered throughout the layer, apparently in relation with the vertical fibres and interstitial connective tissue.

Of the cells, some are of a round and roundly oval form, from $\frac{1}{9000}$ " to $\frac{1}{4500}$ " in diameter. Carmine stains them deeply, and many contain a minute nucleus (figs. 6 \& 7 ). In rather thick sections, in which the relations of the elementary parts are less disturbed, these cells are often seen disposed in linear series parallel to the oblique fibrillation to be presently described. Where the layer is broad, they are most numerous in its middle third. There are other cells which are much larger and of an irregular figure (figs. 8, 9, \& 10). These have a faint single outline, a pale, finely granulated texture, and contain a large, round, sharply-defined nucleus which nearly fills them, averaging $\frac{1}{3000} "$. They occur principally near the inner surface of the layer, and are less numerous than the smaller cells. I have frequently seen a broad, flat, pale 
process pass inwards from these large multiangular cells into the granular layer, and here break up into very fine parallel fibres, which I have traced nearly to the ganglionic layer, and believe to be continuous with processes from the cells of this layer. On the outer side the cells are connected with the oblique fibres.

These fibres (figs. 7, 8, 9, 10, \& 11) are directed obliquely from the outer to the inner surface of the layer, and from the centre towards the periphery of the retina. Their obliquity diminishes towards the inner surface of the layer, where many of them are nearly horizontal. In the outer part of the layer they are very fine and numerous, in the middle they are thicker and fewer, while in the inner third their numbers still further decrease, and they become stouter and flatter. This results from the union of the primitive fibres in bundles, which have an open plexiform arrangement. In the thin periphery of the retina the whole course of the fibres is more horizontal.

The following are the relations of the oblique fibres and the other tissues. The finest fibres in the outer part of the layer are continuous through the intergranule-layer with the primitive fibres of the cone-fibre plexus, as I have already mentioned in describing this; they also take up the smaller round and roundly oval inner granules. Combining as they pass through the layer, becoming stouter and more horizontal, they join the large ganglion-cell-like cells which lie near the inner surface of the layer, and the pale bandlike fibres which these cells send into the granular layer may be regarded as their further continuations.

5. The Granular Layer.-Around the fovea the thickness of this layer nearly equals that of the inner granules; from this point it very gracually decreases towards the periphery of the retina. Its minute structure is a close and intricate web of exceedingly fine soft filaments. It is marked by horizontal parallel bands, which appear to result from a longitudinal disposition of the filaments.

Two sets of rertical fibres are distinguishable in it. The first are connective fibres passing outwards from the membrana limitans interna; they are finer near the centre than at the periphery of the retina. They divide at acute angles, and give off fine lateral branches which are lost in the proper tissue of the layer.

The second set consists of the fibres which pass inwards from the large multipolar inner granules (which I look upon as ganglion-cells), and of those passing outwards from the cells of the ganglionic layer. I have already said that I believe these fibres are continuous. I have occasionally seen large ganglionic cells in vacuoles in the middle of this layer, at a distance from the inner granules and ganglionic layer.

6. The Ganglionic Layer.-The cells of this layer are distinctly multipolar (fig. 12). They have a faint single outline, a soft, finely granulated texture, and a very conspicuous large circular nucleus. On the one side they send off the fibres mentioned in the account of the granular layer, while on the other they send fibres, which cannot be distinguished from nerve-fibres, to the plexiform expansion of the optic nerve. Around the fovea they lie four or five deep; their numbers increase for about $\frac{1}{2}{ }^{\prime \prime \prime}$ from this in a peripheral direction, and then decrease. 
7. Optic Nerve-layer.-The general appearances of the optic nerve have been already described; its finer distribution does not exhibit any special characters. The bundles are disposed in a plexus, the meshes of which transmit the connective radial fibres.

The plexus is separated from the membrana limitans interna by a narrow band, which has a finely granular and striated texture. Its minute structure agrees with that of the granular layer. This band is bounded by the membrana limitans interna, from the inner surface of which the connective radial fibres arise by fine converging roots; their course has been given in the descriptions of the different layers.

\section{Fovea Centralis.}

Owing to the great thickness of the retina immediately around it (fig. 13), the fovea is encircled by a raised margin, from which the surface rapidly declines centrally to the bottom of the fovea, while it slopes gently towards the periphery of the retina. Opposite its margin the optic nerve, granular, inner granule, layers, cone-fibre plexus, and the inner limit of the outer granule-layer, begin to bend outwards, and becoming gradually thinner, they converge to the bottom of the fovea, where they cease, the cones (with their fibres diverging on all sides towards the outer granule-layer) alone, covered by a thin fibro-granular band, existing at this spot.

Reviewing the layers at the fovea in order, from the inner surface of retina outwards, we find the optic nerve-fibres in decreasing number from the margin to the bottom of the fovea.

The ganglion-cells around the bottom lie in a single and interrupted series, which, midway towards the margin, becomes continuous, and double or treble, while at the margin the cells lie four or five deep. The greatest development of the ganglionic layer is not, however, here, but about $\frac{1}{2}{ }^{\prime \prime \prime}$ from the centre of the fovea.

The granular layer blends in the bottom of the fovea with the granular band, which in other parts lies between the optic nerve-layer and the membrana limitans interna. This band in the fovea exhibits a very distinct vertical striation.

The inner granule-layer, in sloping towards the bottom of the fovea, closely approaches the outer granule-layer, as the cone-fibre plexus which lies between them becomes thinner. It attains its maximum development slightly nearer the fovea than that of the ganglionic layer.

The cone-fibre plexus commences near the bottom of the fovea, in the angle betreen the inner and outer granules, and steadily increases in breadth till it reaches its maximum development, slightly nearer the fovea than that of the inner granules.

The outer granule-layer, also commencing near the bottom, swells very rapidly, and opposite the margin of the fovea acquires a remarkable thickness. From this point it slowly declines for about $\frac{3}{4}$ "' from the fovea, and then becomes abruptly thin. Its extraordinary enlargement immediately around the fovea is due to the presence here, not only of the outer granules which belong to the cones lying immediately at its outer surface, but also of those outer granules that belong to the cones which lie at the centre of the 
fovea. Besides which the great proportional number of cones in the fovea is attended with a corresponding increase in the number of the outer granules.

Conal Layer.-The cones become thinner, longer, and more numerous from the periphery to the centre of the retina, i.e. of the fovea. At the centre of the fovea they reach the extraordinary length of $\frac{1}{360} "$. In fresh specimens the characteristic bead in the outer end of the inner segment of the cones here was conspicuous, though very minute.

A careful study of many sections has convinced me that the cones stand vertically on the choroid ; the primitive fibres proceeding from them to the outer granules do, however, incline on all sides from the centre of the fovea; and the bundles of the cone-fibre plexus, as also the oblique fibres in the inner granule-layer, are similarly deflected.

Enumerated briefly, the peculiarities of the fovea consist in the attenuation of the optic nerve, ganglionic, granular layers, and cone-fibre plexus, from its margin towards its centre; in the absence of these layers at the centre; in the great slenderness and length of the cones; in the deflection of the primitive cone-fibres and of their continuations in the outer and inner granule-layers from the centre towards the periphery; and in the maximum development of all the layers, excepting the bacillary, at successively increasing distances from the centre of the fovea in their order of superposition from the outer to the inner surface of the retina.

\section{Explanation of Plate.}

\section{PLATE XIX.}

Fig. 1. An enlarged view of the Fovea centralis in the fresh retina.

Fig. 2. Cones from the Fovea: $a$, the outer; $b$, the inner segment.

Fig. 3. Cones from the periphery of the retina: $a$, the outer segment; $b$, the inner segment containing, $d$, an outer granule, and produced inwards as a primitive fibre through the membrana limitans externa; $c$, intended to mark this latter (the horizontal line), has been placed too high by the artist.

Fig. 4. $A$ vertical section near the Forea parallel to $a$, the bundles of the cone-fibre plexus; $b$, primitive fibres passing from these bundles through $d$, a granular stratum, into the inner granule-layer; $c$, the outer vertically-radial connectivetissue fibres.

Fig. 5. A vertical section through the outer layers near the Fovea. Only the connectivetissue frame is represented: 1, the bacillary layer; 2, the outer granulelayer; 2 ', the cone-fibre plexus; 3 , a granular stratum representing the intergranule-layer; $c$, the membrana limitans externa; $f$, the trunks of the outer vertically-radial connective-tissue fibres; $g$, their outer ends, branching and terminating in the membrana limitans externa (the artist has drawn these too thick); $h$, the branching inner ends of the fibres forming the intergranule- 
layer and terminating in $i$, a thin band bounding the outer surface of the inner granule-layer; $k$, the vertically-radial connective-tissue fibres in the inner granule-layer.

Figs. 6 \& 7. Oblique fibres in the inner granule-layer arranged in a plexiform manner, with smaller inner granules.

Fig. 8. A vertical section through the inner granule-layer, near its inner surface: $a$, larger inner granules (= ganglion-cells ?); $b$, fibres passing from them into the granular layer.

Fig. 9. Two larger inner granules, from the same situation: $a, b$ indicate the same parts as in the preceding figure; $c$, a vertically-radial connective-tissue fibre.

Fig. 10. A vertical section near the inner surface of the inner granule-layer: $a$, smaller; $b$, larger inner granules; $c$, a vertically-radial connective-tissue fibre; $d$, stout band-like (nervous) fibres resulting from the union of the finer oblique fibres, and directed radially from the centre towards the periphery of the retina and from the outer towards the inner surface.

Fig. 11. Two larger (ganglion-cell like) inner granules.

Fig. 12. Ganglion-cells and optic nerve-fibres.

Fig. 13. A vertical section through the Fovea, $\times 20: 1$, bacillary layer (at its outer surface the choroid); owing to a slight artificial foiding of this layer the cones are represented as decreasing slightly in length from the edge to the centre of the fovea instead of increasing as they actually do ; 2 , the outer granulelayer followed by the cone-fibre plexus; 3 , the intergranule-layer; 4 , the inner granule-layer; 5 , the granular layer; 6 , the ganglionic and optic-nerve layers. 
Digitized by the Internet Archive in 2014 

